\title{
Filmsprache und Filmanalyse in der Medienpädagogik
}

\section{Horst Schäfer}

\section{Zusammenfassung}

In der Praxis der Filmseminare mit Studentinnen und Studenten hat sich gezeigt, dass diese meist nicht über hinreichendes Vorwissen für Filmanalysen verfügen und innen auch die Fachbegriffe im Bereich der filmischen Gestaltungsmittel fehlen. Da die Filmanalyse-Seminare allein schon wegen der jedes UniversitätsZeitraster sprengenden Filmlänge sinnvollerweise als Blockveranstaltungen durchgeführt werden, bekommen die Studenten zur Vorbereitung und als Hilfsmittel für eigene Filmanalysen eine grundständige Einführung. Dieses Basiswissen zur "Filmsprache und Filmanalyse in der Medienpädagogik" ist in den letzten zwanzig Jahren immer wieder optimiert worden und wird in der vorliegenden Fassung an der TU Dresden und der Universität Duisburg/Essen eingesetzt.

\section{Kontakt}

Horst Schäfer

Horst.Schaefer1@gmx.de

Leiter des Kinder- und Jugendfilmzentrums in Deutschland (KJF) von 1982 bis 2007, freier Autor und Filmpublizist 


\section{Inhalt}

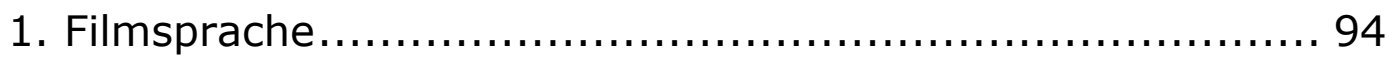

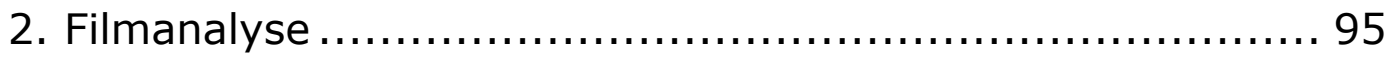

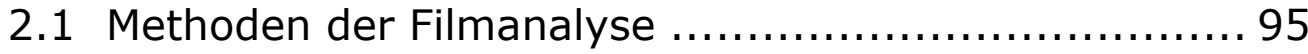

2.2 Die biographische Filmanalyse .......................... 97

2.3 Genrespezifische Filminterpretation .................... 97

2.4 Die soziologische Filminterpretation...................... 97

2.5 Die psychologische Filminterpretation ................... 98

2.6 Die literatur- oder filmhistorische Filminterpretation .... 98

3. Analyse-Modell für medienpädagogische Filmseminare $\ldots . . .100$

4. Medienpädagogische Analyse .............................. 104

4.1. Die emotionale Erlebnisqualität......................... 105

4.2 Die kognitive Erlebnisqualität ......................... 108

4.3 Die ästhetische Erlebnisqualität ....................... 108

4.4 Der Film als potentielles Lernfeld für Kinder bzw. als Kommunikations-Angebot für Jugendliche ............. 109

4.5 Formen der Vor- und Nachbereitung des Films.......... 110

6. Anmerkungen zum Jugendschutz und zur Altersfreigabe

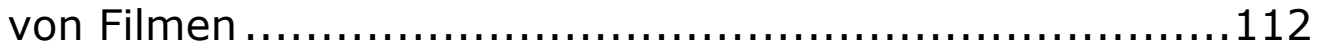

7. Anmerkungen zur Darstellung von Gewalt und zur Medienwirkungsforschung

Literatur 115 


\section{Filmsprache}

Sprache, ikonische Aussagen (Bilder) und kinematographische Bilder sind Zeichensysteme, die bei der zwischenmenschlichen Kommunikation am häufigsten verwendet werden. Die kinematographischen Bilder sind als System - nach Peter Pleyer - durch drei Klassen von Grundelementen gegliedert:

1. Ikonische Zeichen

2. Figuren

3. Kinesische Zeichen (kleinste bedeutungstragende Einheiten in kinetischer Hinsicht)

Das kinematographische Bild ist identisch mit der filmischen Einstellung. Jede Einstellung besteht aus einer Anzahl von Einzelbildern, die erst bei der Projektion des Films als kinematographisches Bild wahrgenommen werden. Jedes dieser Einzelbilder besteht aus ikonischen Zeichen und Figuren. Durch die Projektion entsteht aus dem Übergang vom Einzelbild zur Einstellung die dritte Gliederung: Die Objekte bewegen sich. Diese Objektbewegungen sind kinesische Zeichen.

Aus der dreifachen Gliederung kinematographischer Bilder ergeben sich Konsequenzen für die Filmrezeption: Da Bilder und Objektbewegungen in ihnen wahrgenommen werden, gewinnen die kinematographischen Bilder und damit der Film insgesamt hohe realistische Anmutungsqualität für den Rezipienten; der Film wird als Reproduktion von Wirklichkeit erlebt. Dieser Eindruck wird noch verstärkt durch die gleichzeitige Wahrnehmung von Sprache und anderen akustischen Zeichen und Figuren beim Tonfilm.

Im Film wird eine Vielzahl von kinematographischen Bildern aneinandergefügt. Seine Syntax enthält dementsprechend nicht nur Regeln für die Konstruktion kinematographischer Bilder, sondern auch Regeln für die Montage der Bilder und Regeln für bestimmte filmische Erzählweisen. Semantisch stellt jeder Film (auch jede Fernsehsendung) ein komplexes Bedeutungsgefüge mit erheblicher Bedeutungsmenge dar, die sich ergibt aus: 
- der Bedeutung ikonischer, sprachlicher, kinesischer und sonstiger Einzelzeichen,

- der Kontextbedeutung ikonischer, sprachlicher, kinesischer und sonstiger Einzelzeichen im kinematographischen Bild,

- der Kontextbedeutung kinematographischer Bilder im Gesamtfilm.

Diese Bedeutungsmenge ist in der Regel so groß, dass sie vom Rezipienten nur zum Teil entschlüsselt werden kann.

\section{Filmanalyse}

Die Filmanalyse leistet die Entschlüsselung der Bedeutungsmenge. Ihr Ziel ist es, die Struktur der Filme als auch ihre Wirkung zu untersuchen. Die zentrale Fragestellung ist: Mit welchen Gestaltungsmitteln werden bestimmte Effekte erzielt, die beim Zuschauer bestimmte Wirkungen hervorrufen?

\subsection{Methoden der Filmanalyse}

Zur Analyse eines Films stehen unterschiedliche Verfahrensweisen zur Verfügung, die verschiedenen Disziplinen zugeordnet werden können. Grundsätzlich sind zwei Richtungen der Filmanalyse zu unterscheiden:

a) die empirisch-sozialwissenschaftliche Methode (Inhaltsanalyse), die zum Ziel hat, strukturelle Merkmale auf eine objektivierbare Weise zu ermitteln. Quantitative Inhaltsanalysen werden vor allem im sozial- und publizistikwissenschaftlichen Bereich, aber auch literatur-, kunst- und kommunikationswissenschaftlichen Ansätzen durchgeführt, in denen es beispielsweise auf die Beantwortung von Fragen im Zusammenhang größerer Produktmengen ankommt, so dass sich Häufigkeiten von Merkmalen als ein zusammenfassendes Strukturelement herausstellen lassen. Die ästhetische Struktur des einzelnen Produkts wird dabei eher vernachlässigt. Ein Beispiel dafür ist die ideologie-kritische Inhaltsanalyse der Darstellung von Randgruppen (soziale Außenseiter, kriminelle Jugendliche etc.) in den Medien. Hier sind die engagierte 
Parteilichkeit oder die bewusste Diffamierung ausschlaggebender als die ästhetische Qualität.

b) Der hermeneutische Zugang zu Filmen. Er orientiert sich an der Theorie und Praxis der Textauslegung, indem die vorhandenen Bedeutungsebenen und Sinnpotentiale aufgedeckt werden. Da es um ein Sinnverstehen geht, fließt auch die Subjektivität des Rezipienten in die Analyse ein. Hermeneutisch orientierte Film- und Fernsehanalysten sind sich immer der Historizität der Analyse bewusst.

Die in der Literaturinterpretation entwickelten Arbeitsschritte finden sich auch in der Filmanalyse wieder.

- Ausgehend von der Seh-Erfahrung des Betrachters lässt sich ein erstes Verständnis des Films formulieren, in dem auch Missverständnisse und Nichtverstehen deutlich werden, die zugleich die Subjektivität der Seherfahrungen deutlich machen,

- die eigene Seh-Erfahrung wird als eine spezifische Lesart oder Wahrnehmung artikuliert und zugleich als eine erste Auslegungshypothese (Fragestellung) formuliert.

- Daran knüpft die Analyse des Films an, die sich nicht nur auf subjektive Empfindungen, sondern auf zusätzliche Einsichten stützt, indem durch fachspezifische Methoden die Bedingungen des Filmverständnisses reflektiert werden, die Struktur des Produkts untersucht, seine Ausdrucksformen, seine filmästhetische Gestaltung, seinen Bezug zu den filmischen Traditionen und die in ihm vorhandenen Bedeutungspotentiale entschlüsselt werden.

- Die eigene Seh-Erfahrung, die Lesart des Films und die Analyse der Struktur werden in einem letzten Schritt in einen Zusammenhang gebracht.

Die Filminterpretation kann sich aus verschiedenen Perspektiven auf unterschiedliche Aspekte des Filminhalts beziehen:

Sie stellt das zentrale Gerüst des Films, seinen Aufbau, seine Komposition, seine spezifische Ordnung als Einzelwerk in den 
Mittelpunkt der Untersuchung. Fragen beziehen sich beispielsweise

- auf die Handlung und Handlungsstränge,

- auf die Figuren,

- auf die Stilmittel (Kamera, Bildeinstellungen/Komposition, Montage, Geräusche, Musik),

- auf den Bereich der Werte, Normen, Ideologien.

\subsection{Die biographische Filmanalyse}

Sie zielt nicht auf die Struktur des Films wie beim strukturalistischen Ansatz sondern es geht darum, über den Regisseur und seine Biographie, zu einem besseren Verständnis seines Werkes zu gelangen. An die Stelle der formalen Struktur wird somit eine biographische Struktur gesetzt, beispielsweise die "besondere Handschrift" in den Filmen von Alfred Hitchcock oder David Lynch.

\subsection{Genrespezifische Filminterpretation}

Zu einem Genre zählt eine Gruppe von Filmen, die Gemeinsamkeiten hinsichtlich der Thematik, der Motive, der Symbole, der Handlungsschemata oder auch in Bedeutungen aufweisen. Gängige Genre sind beispielsweise die Love Story, der Thriller, die Teenhorrorfilme oder der Science-Fiction-Film.

Bei der genrespezifischen Filminterpretation werden Filme im Kontext ihres Genres analysiert. Zwei Fragen stehen dabei im Mittelpunkt: Was ist bei dem zu interpretierenden Film unverändert im Verhältnis zu anderen Filmen seines Genres? Was ändert sich im Verhältnis zu den anderen Filmen, welches sind die Variablen, aus denen sich die Genre-Entwicklung ersehen lässt?

\subsection{Die soziologische Filminterpretation}

Hier lautet der Schlüsselbegriff „Gesellschaft”. Die soziologische Filminterpretation geht von einer gesellschaftlichen Prägung des Films aus, insofern als sie den Film im Hinblick auf seine Wiedergabe von Wirklichkeit bewertet. Daraus lässt sich ideologie- 
kritisch die Frage nach der gesellschaftlichen Parteilichkeit des Films ableiten: Parteilichkeit für oder gegen bestimmte Randgruppen, Schichten und Personen, Parteilichkeit in Problemfragen und Interessengegensätzen.

Ein Film kann affirmativ als auch kritisch gegenüber geltenden Normen der Gesellschaft und realen Herrschaftsverhältnissen sein. Es werden Fragen gestellt wie: Wird hier gelogen, verschönt, utopisch ein neuer Weg aufgezeigt? Die Interpretation zielt somit auf die Funktion des Films innerhalb einer bestimmten Gesellschaft.

\subsection{Die psychologische Filminterpretation}

Psychologische Filminterpretationen beschäftigen sich mit Aspekten der Filmwahrnehmung, wobei der Kontext, in den der Film gestellt wird, der Kontext der eigenen Psyche, der Psyche des Zuschauers ist. Das Erkenntnisinteresse besteht darin, aus dem Manifesten eines Films dessen latente Bedeutung zu ermitteln. Vorgängiges Wissen ist beispielsweise die Kenntnis um psychoanalytische Symbole, um Verarbeitungs-, Verdrängungs-, Maskierungs-, Abwehrmechanismen.

Dieser Bereich spielt eine zentrale Rolle in der MedienwirkungsForschung (Darstellung von Gewalt) und im Jugendmedienschutz (psychische Belastung und FSK-Freigaben)

Für die medienpädagogische Einschätzung, d.h. ihre Eignung für Kinder und Jugendliche, bieten sich in erster Linie die strukturalistische, die soziologische und die psychologische Filminterpretation an. Die auf Filmstil oder Filmgeschichte hin ausgerichteten Analysten können das "filmkundliche" Wissen erweitern und vertiefen.

\subsection{Die literatur- oder filmhistorische Filminterpretation}

Das Erkenntnisinteresse dieses Ansatzes zielt auf ein Verständnis des Films im Licht seiner literaturhistorischen oder filmhistorischen Tradition, wie beispielsweise die Serie Noir oder die Nouvelle Vague. Bekanntestes Beispiel in diesem Zusammenhang ist 
die "Literaturverfilmung" nach Vorlagen von Roald Dahl, Astrid Lindgren, Christine Nöstlinger etc.

Für einen aufschlussreichen Vergleich bietet sich der Film "Strangers on a Train" ("Der Fremde im Zug"; USA 1951) an. Die Vorlage ist der Roman von Patricia Highsmith. Das Drehbuch schrieb Raymond Chandler und die Regie führte Alfred Hitchcock.

Ein anderer Zusammenhang ist der explizite oder teils implizite Bezug einen Films auf andere Filme (Filmzitate): Ohne die Kenntnis der Figur des Rick (Humphrey Bogart) in "Casablanca" ist Woody Allens "Mach's noch einmal, Sam" nicht in seiner ganzen Bedeutung zu verstehen.

Aktuelle Beispiele: "Inglourious Basterds" von Quentin Tarantino (D/USA 2009) und "Machete" von Robert Rodriguez (USA 2010).

\section{Literaturverfilmung}

Als Literaturverfilmung bezeichnet man die Umsetzung (filmische Version) einer literarischen Vorlage. Die Ausgangsbasis kann verschiedenen literarischen Gattungen entnommen sein: Roman, Drama, Kurzgeschichte und Erzählung.

"Der etwas diffuse Begriff Literaturverfilmung sollte nur dann verwendet werden, wenn man der literarischen Vorlage zu einem Film hohen Rang zumisst, meist übereinstimmend mit dem geltenden kulturgeschichtlichen Kanon der Meisterwerke". (Reclams Sachlexikon des Films)

Adaptionsformen (nach Wolfgang Gast):

1. Adaption als Aneignung von literarischem Rohstoff. Viele Filme sind geschaffen "nach Motiven" etwa der Abenteuerliteratur.

2. Adaption als Illustration. Diese "bebilderte Literatur" vernachlässigt die Eigengesetzlichkeiten der beiden Medien und bringt meist keine interessanten Transformationen zustande, weil die Vorlage ins Visuelle kopiert wird, was weitgehend zu konventionellen und auch langweiligen Endprodukten führt, besonders bei den Verfilmungen von Kinderbüchern, aber auch in anderen Literaturverfilmungen. 
3. Adaption als Transformation. Hier wird umgesetzt, aber ohne Kopierabsicht, sondern die Verfilmung sucht ein "analoges Werk", die medienspezifischen Bedingungen leiten den Einsatz der Verfahren und die Veränderungen in der Vorlage, denn statt Wörtliches zu zitieren werden effektive Entsprechungen intendiert. Das kann zu interpretierenden Transformationen führen, die auf radikal subjektive Weise die Vorlage verändert, aber sich dennoch an deren Geist orientiert und dem Zuschauer die Verschiedenheit von Vorlage und Verfilmung, d. h. den interpretierenden Standpunkt bewusst macht.

4. Adaption als Dokumentation. Hier handelt es sich vor allem um die Verfilmung von Theaterinszenierungen, die die filmischen Mittel so einsetzen können, dass filmgerechtere Aufzeichnungen entstehen.

5. Inhaltlichem Konzept nach unterscheidet Gast noch die aktualisierende, aktuell-politisierende, ideologisierende, historisierende, ästhetisierende, psychologisierende, popularisierende und parodierende Adaption; Differenzierungen, die je nach Inhaltsgesichtspunkt modifiziert werden können.

\section{Analyse-Modell für medienpädagogische Filmseminare}

Erinnert sei an die zentrale Fragestellung: Mit welchen Gestaltungsmitteln werden bestimmte Effekte erzielt, die beim Zuschauer bestimmte Wirkungen hervorrufen? Ausgangspunkte der Analyse sind die strukturellen Elemente der Filmhandlung und die filmspezifischen Gestaltungsmittel der Handlungsdarbietung. Das nachfolgende Analyse-Modell orientiert sich in Gegensatz zum Stellenwert an einer Filmakademie hier an dem Gebrauchswert für die Medienpädagogik - es werden daher nur die wesentlichen und gebräuchlichsten Begriffe und Elemente einbezogen.

\section{Zur Struktur: Inhalt und Handlung des Films}

- Thema

- Hauptperson

- Nebenrollen 
- Handlungsmotivation

- Konflikte/Konfliktlösungen

- Dramaturgie (Szenenfolge, Höhepunkte, Spannungsbogen)

- Erzählperspektive

- Besondere Gestaltungsmittel (Rückblenden, Traumsequenzen, Collagen etc.)

\section{Zur Technik: Filmsprache}

Zwischen extremer Nähe und extremer Entfernung hat sich eine achtstufige Skala von Einstellungsgrößen eingebürgert - so Wolfgang Gast in seiner "Einführung in Begriffe und Methoden der Filmanalyse" -, die von der Produktionsseite her nötig sind und zugleich auch für eine differenzierte Analyse hilfreiche Kategorisierungen anbieten:

\section{Einstellungsgrößen}

- Weit - Atmosphäre, Spannung, Entspannung

- Total - Handlung im Gesamtüberblick, Übersicht, räumliche Orientierung

- Halbtotal - Distanz zum Zuschauer, sichtbare Körpersprache, jedoch keine Mimik

- Halbnah - Situation, Kommunikation, beobachtende Distanz

- Amerikanisch - "Personen etwa bis unterhalb der Hüfte" (sodass im Western der Colt noch im Bild ist...)

- Nah - Mimik, Gestik, emotionale Nähe

- Groß - Kopf: Ausdruck von Gefühlen und Empfindungen

- Detail - extrem kleiner Ausschnitt, Intensivierung, Spannung

\section{Kameraperspektiven}

- Normalsicht - Augenhöhe (der Erwachsenen, der Kinder)

- Froschperspektive - Untersicht (übermächtiges Gegenüber, lächerlich)

- Vogelperspektive - Aufsicht (geringschätziges Gegenüber, überlegen) 
Die Kameraperspektive spielt besonders eine Rolle bei der medienpädagogischen Einschätzung eines Films hinsichtlich der Eignung für Kinder, siehe Abschnitt 2.3.): Entspricht die gewählte Grund-Perspektive (Normalsicht) der Augenhöhe der Kinder oder wird ihre Wahrnehmung durch die Perspektive eines Erwachsenen verzerrt wiedergegeben?

\section{Kamera- und Objektbewegungen}

- Kamerabewegungen - Stand, Schwenk, Fahrt, Zoom

- "Subjektive" Kamera - Authentizität, Identifikation

- Kamera- und Objektbewegungen

a) in das Bild hinein und vom Zuschauer weg

b) aus dem Bild heraus und auf den Zuschauer zu

c) parallel zum unteren Bildrand von rechts nach links oder umgekehrt am Zuschauer vorbei

Zum Achsensprung (Crossing the Line) kommt es bei Nichteinhaltung der sogenannten 180-Grad-Regel. Diese legt fest, dass die Filmkamera innerhalb eines Halbkreises auf einer Seite der Handlungsachse aufgestellt bleiben muss, damit Bewegungen für die Zuschauer in gleicher Blickrichtung erfolgen. Wird die Achse überschritten, haben die Zuschauer den Eindruck, plötzlich aus entgegengesetzter Perspektive zu schauen, was ihre Orientierung im filmischen Raum erschwert. Der Achsensprung (in der Regel ein Fehler) kann auch absichtsvoll eingesetzt werden wie beispielsweise im Monolog von Gollum bzw. Smeagol („Herr der Ringe: Die Gefährten, 2001), um die innere Zerrissenheit und Widersprüchlichkeit der Figur zu zeigen.

\section{Beleuchtung und Farbgestaltung}

- Ausleuchtung, Hervorhebung, Kontraste (Stimmungen)

\section{Wort-Bild-Ton-Beziehung}

- "On" - sichtbare Quelle

- "Off" - unsichtbare Quelle

- Parallel-synchroner Ton (aktueller Ton, kommentierender Ton) 
- Kontrapunktisch-synchroner Ton (Unterschiedliche Semantik)

\section{Musik}

- illustriert, kommentiert (Handlung, Stimmungen)

- etabliert (Raum und Zeit)

- emotionalisiert (Filmrezipienten)

- strukturiert (Kontinuität, Zäsur)

- Titel- und Leitmotiv (Kanonisierung, Werbung)

\section{Geräusche}

- Intensivieren (authentisch, synthetisch)

\section{Special effects}

- Filmtricks, Rückprojektionen, Digitalisierung

\section{Computer Generated Imagery (CGI)}

Fachausdruck für mittels 3-D-Computergrafik erzeugte Bilder im Bereich der Special effects (auch: Visual Effects). Der Begriff bezeichnet Computeranimationen in der Filmkunst - im Gegensatz zu Computeranimationen zum Beispiel in Computerspielen.

\section{Dokumentarfilm}

Umfassender, allgemeiner Begriff für alle nichtfiktionalen Filme in unterschiedlichen Formen wie "semi-dokumentarisch" für Spielfilme, die sich den Anschein eines Dokumentarfilms geben beispielsweise in Filmen über aktuelle Kriegsschauplätze - , oder die "dokumentarische Rekonstruktion" (auch: „Dokudrama"), die beispielsweise ein historisches/zeitgeschichtliches Ereignis in Form eines Spielfilms möglichst authentisch darstellt.

\section{Montage}

- Die Montage ist die Verknüpfung von mindestens zwei Einstellungen oder ganzer Sequenzen eines Films durch Schnitt (cutting) oder Blende. Unterschieden wird dabei zwischen dem sichtbaren, gestaltenden Schnitt und dem unsichtbaren, weichen Schnitt.

Die sieben Grundtypen der Montage sind: 
- Szenische Montage (Augenzeuge, Einheit von Raum, Zeit und Handlung)

- Erzählende Montage (inhaltliche Einheit, zusammenhängender Prozess)

- Beschreibende Montage (objektbezogen; Schauplätze, Gegenstände und Figuren)

- Metonymische Montage (umfassende Klammerung; die Addition einzelner Teile ergibt ein Neues)

- Vergleichende Montage (pointiert aneinander geschnitten und miteinander verbunden)

- Symbolische Montage (Symbole: Uhr = vergehende Zeit/Wildpferde $=$ Freiheit und Abenteuer)

- Assoziative Montage (Vermittlung verschiedener Wirkungsebenen, Atmosphäre, Impressionen)

\section{Medienpädagogische Analyse}

Der Analyse des Films folgt die Einschätzung des Films hinsichtlich der besonderen (medienpädagogischen) Einschätzung für Kinder und Jugendliche. Es wird davon ausgegangen, dass die Auswahl der Filme für Kinder von den Medienpädagogen direkt verantwortet wird und möglicherweise nur ein Teil des pädagogischen Programms (Thema - Zielsetzung - Vorbereitung Film - Nachbereitung) ist, während Jugendliche stärker in die Auswahl einbezogen werden und es hier sehr stark auf eine Kommunikation anregende, dialogstiftende Funktion ankommt.

Die Frage nach der Eignung von Filmen für Kinder (Zielgruppe 6 bis 12 Jahre) und Jugendliche (Zielgruppe 12 bis 16 Jahre) lässt sich grundsätzlich auf folgende vier Dimensionen beziehen:

- Die emotionale Erlebnisqualität

- Die kognitive Erlebnisqualität

- Die ästhetische Erlebnisqualität

- Der Film als potentielles Lernfeld für Kinder bzw. als Kommunikations-Angebot für Jugendliche 
Diesen vier Dimensionen können folgende Kriterien zur Einschätzung zugeordnet werden:

\subsection{Die emotionale Erlebnisqualität}

a) Betroffenheit und Identifikation

Die Grundannahme ist: Kinder haben eine hohe Bereitschaft zum emotionalen Miterleben und zur Identifikation. Das Spektrum des altersspezifischen Rezeptionsvermögens reicht vom ,totalen' Erleben bei den Vorschulkindern bis zur emotionalen Distanzierung bei den älteren Kindern. Das Ausmaß von tatsächlicher Erlebensintensität und Identifikation hängt $a b$ von der Nachvollziehbarkeit der Situationen und der Handlung des Films, von der dramaturgischen Struktur und vom Vorhandensein von Identifikationsfiguren.

Filme, deren Handlung (die erzählte Geschichte oder einzelne Geschehnisse) die Kinder betroffen macht oder deren Protagonisten - beispielsweise gleichaltrige Kinder - zur Identifikation einladen, sind besonders gut für die Kinderfilmarbeit geeignet.

Dieser Prozess verläuft über die Projektion (eigene Wünsche und Vorstellungen werden in das Geschehen projiziert) und die Introjektion (unbewusste Nachahmung oder Einbeziehung fremder Anschauungen/Motive in den eigenen Interessenkreis).

Kinder identifizieren sich aber nicht nur mit den "Filmkindern", den Protagonisten, sondern auch mit Tieren als handlungstragende Figuren; besonders bei jüngeren Kindern sind diese sehr beliebt. Schwierig ist es bei Filmen, in denen aus dramaturgischen Gründen anfangs bewusst, negative' Protagonisten aufgebaut werden - etwa um Klischee-Charaktere wie beispielsweise unbeliebte Außenseiter später zu brechen und sie sympathisch werden zu lassen. Das Interesse der Kinder an der Entwicklung dieser Figuren ist nicht besonders ausgeprägt.

Auch Jugendliche identifizieren sich in erster Linie mit den positiven Protagonisten: mit den Stars, Idolen und Ikonen des populären Kinos. Die Filmbranche produziert unterschiedliche Idole, und sie lösen einander schnell ab. Während der Star als Gegenstand der Bewunderung aller noch eine gewisse kulturindustriell ver- 
mittelte Verbindlichkeit ausdrückt, muss das Idol von denen ergriffen werden, die sich mit ihm ein Stück weit identifizieren können - sonst verliert es Kontur und Magie. Schauspieler und gespielte Figur werden zunehmend untrennbar. Sie "spielen sich selbst" und werden damit von Idolen zu Ikonen. Die neuen jugendlichen Ikonen sind keine Verwandlungskünstler mit einer Allmacht über diverse Gesten und Rollenrepertoires, sondern sie sind stets dieselben, für jeden wiedererkennbar und gerade darum geliebt, verehrt, und wirksame Identifikationsobjekte (Bei-spiele: früher die Stars James Dean, Marlon Brando, Romy Schneider und Audrey Hepburn; heute sind es mehr ,Charaktere' wie Harry Potter und Katniss Everdeen oder auch ,Swashbuckler' wie Captain Jack Sparrow).

Dazu Dieter Baacke in "Leben wie im Kino" (1994):

"Die neuen Jugend-Idole sind Delegierte. Sie werden von ihren Zuschauern ,ausgeschickt', um jene Grenzerfahrungen zu machen, jene Lebensversuche zu formulieren, von denen ihre jugendlichen Zuschauer träumen. Der Alltag der meisten Jugendlichen lässt nicht zu, was der Film verdichtet zeigt: action, body, emotion. Zwischen den JugendIdolen und ihren Zuschauern besteht eine Psychodynamik gegenseitigen Angewiesenseins. Der Delegierte tut, was der andere tun möchte, aber nicht zu tun sich getraut.

Wichtig am Idol ist zwar seine Authentizität, die Stimmigkeit dessen, was es als Delegierter für seine Zuschauer erlebt und ausführt. Aber es wächst sozusagen über den Rand des (imaginären) Filmgeschehens hinaus, und so wird die Trennlinie unscharf, die filmische Fiktion und reales Leben eindeutig unterscheidbar machen."

Identifikationsprozesse finden auch und gerade dort statt, wo nicht die Stars und Idole - beispielsweise des synthetischen Hollywood-Kinos - die Protagonisten sind, sondern Jugendliche in authentischen Filmen. Das sind Filme, die als zeitliche und geographische Gemeinsamkeiten aktuelle Bezüge zur Alltagsrealität der Jugendlichen aufweisen und ihrem Lebensgefühl entsprechen. In den Angeboten zur Identifikation mit den Protagonisten und zur Betroffenheit von dem Geschehen besitzen diese Filme 
ihre thematische und motivische Gemeinsamkeit. Authentische Produktionen machen sich die Perspektive der Jugendlichen zu eigen. Durch Parteilichkeit und Nähe unterscheidet sich dieser Ansatz beispielsweise von den Filmen, die auf eine voyeuristische Distanz zu Jugendlichen gehen - sehr oft dort, wo Problembereiche wie Jugendkriminalität, Banden, Drogen, Prostitution etc. kommerziell ausgeschlachtet werden.

Auch Filme ohne Identifikationsfiguren können Betroffenheit auslösen und Jugendliche faszinieren. Ein Beispiel hierfür sind Filme, die den "Geist der Zeit" (oder einer bestimmten) treffen wie beispielsweise der experimentelle Dokumentarfilm "Koyaanisqatsi" (USA 1976-1982); ein Film über den Missbrauch der Erde durch den Menschen und die Schädigung urbaner Zivilisation, eine Montage von Bildern und Musik ohne ein gesprochenes Wort.

b) Psychische Belastung durch bestimmte Inhalte

Die Grundannahme ist: Filme, in denen bestimmte bedrohliche Situationen besonders häufig vorkommen, sind für Kinder zu stark psychisch belastend. Eine nicht altersgerecht-zumutbare psychische Belastung spricht gegen den Einsatz eines Films. Hierzu gehören ggf. beispielsweise die Verlassenheit von Personen/Kindern in fremder Umgebung, angstauslösende Situationen, plötzlich auftretende, erschreckende Ereignisse und traurige Situationen.

Für die Medienpädagogik ist die spezifische Eignung des Films beispielsweise für die Aufbereitung eines Themas in der Kinderfilmarbeit ausschlaggebend. Vorausgesetzt wird hierbei die Altersfreigabe des Films durch die Freiwillige Selbstkontrolle der Filmwirtschaft (FSK), die nicht unterschritten werden darf. Die gesetzliche Freigabe markiert eine untere Grenze unterhalb der das Risiko psychischer Verletzungen und Entwicklungsstörungen besteht. Dieser Grenzwert wird häufig als pädagogische Altersempfehlung fehlinterpretiert. 


\subsection{Die kognitive Erlebnisqualität}

Hierzu zählen inhaltliche und formale Eindeutigkeit und Einfachheit: Die Verständlichkeit der Geschichte und des Verhaltens der Personen, der inneren Entwicklungen sowie möglicher Rückblenden oder Traumsequenzen, sowie eine überschaubare Erzählstruktur, Klarheit in den Personenkonstellationen, sozialen Beziehungen oder Entwicklungen.

Geschehens- und Sinnverständnis eines Films sind altersabhängig. Je eindeutiger und einfacher ein Film inhaltlich und formal ist, umso besser ist er für jeweils jüngere Kinder verstehbar.

Die kognitive Filmwahrnehmung ist nach den lerntheoretischen Entwicklungsstufen (altersspezifische Intelligenzentwicklung) in verschiedene Stadien (4-6 J., 7-12 J., ab 12 J.) einzuteilen.

Der Einschätzung der kognitiven Erlebnisqualität hinsichtlich der altersspezifischen Eignung geht die Analyse der filmsprachlichen Mittel voraus (s.o.).

\subsection{Die ästhetische Erlebnisqualität}

Die Vermittlung bzw. Aneignung von Medienkompetenz versteht sich auch als offener Prozess ästhetischer Bildung. Zur Qualifikation der Medienrezipienten tragen die Entschlüsselung, das Verständnis und die Deutung der audiovisuellen Produktionen bei.

Erkenntnisse hierzu ergeben sich aus der Analyse des Films (vgl. Abschnitt 2.3.) und der Folgerungen hinsichtlich der künstlerischen und dramaturgischen Gestaltung: Darstellung, Regie, Ausstattung, Special Effects etc.

Um bei Jugendlichen Akzeptanz zu finden, müssen die Filme ihren Sehgewohnheiten entsprechen. Das stellt einerseits hohe Anforderungen an die ästhetische Qualität, schließt aber andererseits nicht aus, jugendliche Zuschauer immer wieder neu herauszufordern. In produktionstechnischer Hinsicht hat es sich für die Akzeptanz bei Jugendlichen als erfolgreich erwiesen, nicht nur mit Profis und Stars, sondern so viel wie möglich auch mit jugendlichen Darstellern direkt zu arbeiten. Laiendarsteller wirken 
als jugendliche Protagonisten oft überzeugender als junge Schauspieler oder Jungstars, die zwar äußerst populär sind, aber nicht mit ihren Rollen identifiziert werden (Beispiel: Winona Ryder, Leonardo DiCaprio). Einen authentischen Bezug - beispielsweise mit Jugendlichen aus einem bestimmten Milieu oder mit einem der Filmhandlung entsprechenden Erfahrungshintergrund - erleichtern den Identifikationsprozess und erhöhen den Grad der Betroffenheit (siehe oben).

\subsection{Der Film als potentielles Lernfeld für Kinder bzw. als Kommunikations-Angebot für Jugendliche}

Filme können für Kinder als Sozialisations- und Erziehungsinstanz bedeutend sein. Dieses erschließt sich über

- Personenkonstellationen und soziale Beziehungen:

Entwicklung sozialer Aggregate - Einzelne/Gruppen (Familie), Menge/ Masse - Beziehungen und ihre Begründungen

- Konflikte und Konfliktverhalten:

Anlässe und Gewichtung (Art), Beteiligte und Konfliktverhalten (emotional/rational, verbal/nonverbal, gewaltlos/mittels Gewalt), Konfliktlösungen (Einlenken eines/aller Beteiligten, "Kompromiss" oder "Sieg"?), Eingreifen anderer Personen bzw. einer höheren Gewalt/Instanz. Übertragbarkeit von Konfliktlösungen, Lösungsmöglichkeiten oder "offenes Ende"? Sind andere Lösungen als die durch Gewaltanwendung denkbar?

- Werte und Wertorientierungen und deren Beurteilung nach eigenen pädagogischen Normen:

Jeder Film vermittelt die Erfahrung einer fiktiven Wirklichkeit, die - insbesondere von jüngeren Kindern - als solche nicht erkannt wird. Entsprechend ausgeprägt ist die Bereitschaft, aus der filmischen Welterfahrung zu lernen und Informationen über die Realität (Fakten- und Wertewissen) abzuleiten. Das Ausmaß, in dem im Einzelfall tatsächlich aus einem Film gelernt wird, ist von der beim Filmempfang vorhandenen Prädisposition abhängig. 
Für Jugendliche sind in der medienpädagogischen Arbeit besonders diejenigen Filme geeignet, die eine dialogstiftende Qualität besitzen und Kommunikationsprozesse initiieren. Das erfordert:

- Identifikationsfiguren

- Glaubwürdigkeit durch die (parteiliche) Sicht der Jugendlichen

- Keine plakative und platte, sondern eine einfühlsame, feinsinnige und liebevolle Dramaturgie

- Keine abgehobene abstrakte, sondern eine nachvollziehbare und lebensnah gestaltete Handlung

- Keine reaktionären und (bloß angepassten) normgerechten Verhaltensweisen, sondern phantasievolle, bewusstseinserweiternde und innovative.

- Nicht verbietende Lösungen oder moralische Zeigefinger, sondern Lösungen oder Lösungsansätze, die man überdenken kann. Besonders hierzu sind Filme mit einem "offenen Ende" ideal.

\subsection{Formen der Vor- und Nachbereitung des Films}

Aus den „potentiellen Lernfeldern" für Kinder (siehe oben) lassen sich die Themen für vielfältige Formen der Vor- und Nachbereitung von Kinderfilmen entwickeln. Ausgangspunkt dieser Arbeit war die Wiederentdeckung des Kinderfilms Anfang der 1970er Jahre. Der Kinderfilm wurde als wichtiger Teil der Kinderkultur anerkannt und das Kinderkino als sozialer, kultureller, pädagogischer und ästhetischer Ort entdeckt und geschätzt. Damit waren auch die Voraussetzungen für eine differenzierte medienpädagogische Vor- und Nachbereitung geschaffen worden. Ausgangsund Mittelpunkt der Aktivitäten ist das Medium Film. So gibt es eine Reihe von Produktionen, die keine Nachbereitung brauchen, die in ihrer Bedeutung, ihrer Wirkung und als Erlebnis für sich stehen. Das schließt jedoch bei anderen Werken eine sinnvolle Vor- und Nachbereitung, die den Film ergänzt und nicht ersetzt, nicht aus.

In dem Sonderdruck "Inspiration Kinderfilm" (1995) der "Kinderund Jugendfilmkorrespondenz" werden in der Praxis erprobte Modelle der Vor- und Nachbereitung exemplarisch dokumentiert, 
die die Wahrnehmungsfähigkeit der Kinder fördern und den kompetenten Umgang mit Bildern und Symbolen entwickeln helfen. Hans Strobel führt in seiner Einleitung folgendes Spektrum auf:

"1. Vor dem Film

Eine Einführung bzw. ,Ansage' zum Film kann folgende Punkte enthalten:

- Informationen zum besseren Verständnis des Films, d.h. Informationen, die sich aus dem Film nicht erschließen, aber zum Gesamtverständnis notwendig sind, z.B. bei zeitgeschichtlichen Filmen oder Filmen aus fremden Ländern oder fremden Kulturen,

- Informationen über die Filmemacher, über die Autoren, z.B. bei Kinderbuchverfilmungen,

- Informationen, die die anschließenden Aktionen betreffen bzw. vorbereiten,

- Einstimmungen, die das Filmerlebnis emotional vorbereiten.

\section{Nach dem Film}

Es gibt eine Vielfalt erprobter Nachbereitungsaktionen; teilweise werden auch ihre verschiedenen Formen miteinander verknüpft.

- Sprachliche Methoden:

Dazu zählen verschiedene Formen des Filmgesprächs und der Filmanalyse - beispielsweise im Schreiben von Kritiken -, die nur in besonderen Zusammenhängen Anwendung finden.

- Spielerische oder spielpädagogische Methoden:

Ein Schwergewicht liegt auf den non-verbalen, den spielerischen Formen und Möglichkeiten. Hier ist vor allem das Rollen- und Theaterspiel zu nennen. Im Spiel können die Kinder die Personen oder Figuren, mit denen sie sich besonders identifiziert haben, nachspielen, neue 
Handlungs- und Lösungsmöglichkeiten entwickeln oder ihre Gefühle verarbeiten.

- Mediale Methoden:

Gemeint sind mediale Angebote und Formen wie z.B. Malen, Collagen erstellen sowie Basteln. Interessant ist auch das Filmen mit der Videokamera. Hier lernen die Kinder, mit einer Kamera umzugehen, die Wirkung diverser Einstellungsarten auszuprobieren oder - angeregt durch den Film - einen eigenen kleinen Film zu drehen. Zur Vermittlung von Grundbegriffen der bewegten Bilder bietet sich neben der Videoarbeit die Direktbearbeitung von Filmmaterial (Blank- und Schwarzfilm) an. Die Welt der Bilder durchschaubarer zu machen, ohne zu entzaubern, ist das Ziel der Beschäftigung mit dem optischen Spielzeug aus der Vorgeschichte des Films. Daumenkinos, Wunderräder, Zwirbelkärtchen etc. lassen sich mit Vergnügen herstellen und sind zugleich aufschlussreiche, faszinierende Schauobjekte."

Das „Lexikon des Kinder- und Jugendfilms" stellt in „Teil 9: Kinopraxis" eine Reihe von medienpädagogischen Vor- und Nachbereitungsideen vor, die sich an einzelnen Filmen oder Filmgenres orientieren und in der Praxis erprobt wurden. Hier werden neben den klassischen, filmgeschichtlich bedeutsamen Filmen auch aktuelle Produktionen mit einbezogen, die in den Medienangeboten für Kinder für nichtkommerzielle Zwecke nutzbar sind.

\section{Anmerkungen zum Jugendschutz und zur Altersfreigabe von Filmen}

„Die FSK-Kennzeichnungen erfolgen auf der Grundlage von §§ 12,14 Jugendschutzgesetz.

Sie sind gesetzlich verbindliche Kennzeichen, die von der FSK im Auftrag der Obersten Landesjugendbehörden vorgenommen werden.

Die FSK-Kennzeichnungen sind keine pädagogischen Empfehlungen, sondern sollen sicherstellen, dass das körperliche, geistige 
oder seelische Wohl von Kindern und Jugendlichen einer bestimmten Altersgruppe nicht beeinträchtigt wird." (Verpflichtender Kennzeichnungstext im Vorspann jeder veröffentlichten DVD)

Mit der Altersfreigabe ist also keine pädagogische Empfehlung oder ästhetische Bewertung verbunden.

Zu den „Pädagogischen Altersempfehlungen für Kinderfilme" hat das "Kinder- und Jugendfilmzentrum in Deutschland" (KJF) 2011 eine Expertise herausgegeben, die auf der Internetsite des KJF downloadbar ist.

\section{Anmerkungen zur Darstellung von Gewalt und zur Medienwirkungsforschung}

"Die Allgegenwart von Gewaltdarstellungen in den Medien und die Frage ihrer Wirkung sind als Themen der Medienpädagogik ein Dauerbrenner"

(Ralf Vollbrecht)

Jugendliche verfügen bereits über eine ausgeprägte Mediensozialisation, was sich beispielsweise in ihren ausgesprochenen Vorlieben für Actionfilme oder aktuell der Popularität der Teenhorrorfilme ausdrückt. Zu den möglichen Gefährdungspotentialen eines Films zählt hier in erster Linie die Darstellung von Gewalt. Verschiedene Modelle der Medienwirkungsforschung (z.B. Katharsisthese, Stimulierungsthese oder Habitualisierungsthese) kommen zu unterschiedlichen Aussagen. Unbestritten ist, dass Gewaltverherrlichung mit einem Wirkungsrisiko verbunden ist. Mit der immer selbstverständlicheren Präsenz von audiovisuellen Medien im Alltag von Kindern und Jugendliches ist dieses Thema von zunehmender Brisanz.

Ralf Vollbrecht in seiner "Einführung in die Medienpädagogik":

„Was aber wird (nicht nur von Kindern) überhaupt als Gewalt wahrgenommen? Ob eine Handlung als Gewalthandlung (soziale Abweichung) oder als normal angesehen wird, unterliegt der Deutung. Es gibt keine Gewalt an sich - weder in den Medien noch in der Realität. Deutungsmuster 
entscheiden darüber, was als Gewalt angesehen wird und welche Gewalt negativ oder positiv sanktioniert oder gerechtfertigt ist (Notwehr). Solche Deutungsmuster finden inre juristischen Formen im Strafgesetzbuch. Wesentlicher als das konkrete Ausmaß an Gewaltanwendung ist die durch Normen und moralische Vorstellungen gestützte soziale Kontrolle. Der soziale Kontext ist dabei ebenfalls von entscheidender Bedeutung... Es gibt also neben Normen und Rechtfertigungen immer auch Ursachen oder Motive für Gewalthandlungen, ebenso Folgen, die selbst wieder Gewalthandlungen sein können. Gewaltdarstellungen müssen daher im Rahmen der Erzähl- und Handlungskontexte gesehen bzw. gedeutet werden. Auch die Genres spielen eine Rolle, da sie die Vorerwartungen der Zuschauer steuern. $(\ldots)$

Bei personaler Gewalt ist auch eine mögliche Identifikation mit dem Täter zu bedenken. Mögliche Motive wären hier Selbstbehauptung, Selbst-Durchsetzung oder Identifikation mit dem Aggressor. (...)

Wenn Gewaltwirkungen der Medien pauschal nicht nachweisbar sind, aber Indikatoren für ein Wirkungsrisiko bei entsprechender individueller oder sozialer Disposition vorliegen, ist für die Praxis der Medienerziehung wohl anzuraten, sich auf die sichere Seite zu schlagen und stets mit der Möglichkeit von Wirkungen zu rechnen (Risikothese). Der Begriff des Risikos unterstellt im Unterschied zum Begriff der Gefährdung nicht das Vorhandensein einer generellen Gefahrenlage, schließt jedoch die Möglichkeit ein, dass für bestimmte Individuen oder Gruppen unter bestimmten Bedingungen ein Wirkungsrisiko besteht." 


\section{Literatur}

Albersmeier, Franz-Josef 2001: Texte zur Theorie des Films. Reclam TB 9943, Philipp Reclam Jun., Stuttgart.

Baacke, Dieter/Schäfer, Horst 1994: Leben wie im Kino. Jugendkulturen und Film. Frankfurt.

Barg, Werner/Niesyto, Horst/Schmolling, Jan 2006: Jugend: Film: Kultur. Grundlagen und Praxishilfen für die Filmbildung. kopaed, München.

Becker, Wolfgang/Schöll, Norbert 1983: Methoden und Praxis der Filmanalyse. Leske + Budrich, Opladen.

Beiken, Peter 2004: Wie interpretiert man einen Film? Für die Sekundarstufe II. Reclam TB 15227, Philipp Reclam Jun., Stuttgart.

Bergala, Alain 2006: Kino als Kunst. Filmvermittlung an der Schule und anderswo. Schüren Verlag, Marburg.

Bienk, Alice 2008: Filmsprache. Einführung in die interaktive Filmanalyse. Schüren Verlag, Marburg (orig. 2006).

Blothner, Dirk 1999: Erlebniswelt Kino. Über die unbewusste Wirkung des Films. Bastei Lübbe, Bergisch Gladbach.

Bordwell, David 2001: Visual Style in Cinema. Vier Kapitel Filmgeschichte. Verlag der Autoren, Frankfurt.

Brücks, Arne/Wegener, Claudia (Hg.) 2009: Filmerleben von Kindern und Jugendlichen. Eine HFF-Studie. In: Schäfer, $\mathrm{H}$. $(\mathrm{Hg}$.$) : Lexikon des Kinder- und Jugendfilms. Corian-Verlag,$ Meitingen (Loseblattsammlung).

Decke-Cornill, Helene/Luca, Renate (Hg.) 2007: Jugendliche im Film - Filme für Jugendliche. Medienpädagogische, bildungstheoretische und didaktische Perspektiven. kopaed, München. 
Diederichs, Helmut H. (Hg.) 2004: Geschichte der Filmtheorie. Kunsttheoretische Texte von Méliès bis Arnheim. Suhrkamp Taschenbuch 1652, Frankfurt.

Ehrenspeck, Yvonne/Schäffer, Burkhard (Hg.) 2003: Film- und Fotoanalyse in der Erziehungswissenschaft. Leske + Budrich, Opladen.

Faulstich, Werner 1994: Einführung in die Filmanalyse. Gunter Narr Verlag, Tübingen (4., vollst. neu bearb. u. erhebl. erw. Aufl.; orig. 1980).

Faulstich, Werner/Faulstich, Ingeborg 1977: Modelle der Filmanalyse. Wilhelm Fink Verlag, München.

Faulstich, Werner 1988: Die Filminterpretation. Vandenhoeck \& Ruprecht, Göttingen.

Felix, Jürgen 2002: Moderne Film Theorie. Theo Bender Verlag, Mainz.

Fritz, Karsten/Sting, Stephan/Vollbrecht, Ralf (Hg.) 2003: Mediensozialisation. Pädagogische Perspektiven des Aufwachsens in Medienwelten. Leske + Budrich Verlag, Opladen.

Gast, Wolfgang 1993: Einführung in Begriffe und Methoden der Filmanalyse. Arbeitsbuch zu Film und Literatur. Diesterweg Verlag, Frankfurt am Main.

Hickethier, Knut 1993: Film- und Fernsehanalyse. Stuttgart/ Weimar, Sammlung Metzler.

Hofmann, Anna/Lassacher, Martina, (Hg.) 2013: Kino erleben und begreifen. Filmanalyse mit Kindern und Jugendlichen. Facultas Verlag, Wien.

Hohmann, Tanja 2002: Medienkompetenz und Kinderkino Modelle der medienpädagogischen Vor- und Nachbereitung. Sonderdruck der Kinder- und Jugendfilm Korrespondenz $(\mathrm{KJK})$. Herausgeber: Kinderkino München e.V./ Kinder- und Jugendfilmzentrum in Deutschland. München (zu beziehen über http://www.kjk-muenchen.de/archiv/sonderdrucke. php). 
Hönge, Volker 1999: Freiwillige Selbstkontrolle der Filmwirtschaft (FSK). In: Horst Schäfer (Hg.): Lexikon des Kinderund Jugendfilms im Kino, im Fernsehen und auf Video. Corian-Verlag, Meitingen (Loseblattsammlung).

Kinderkino München e.V./Kinder- und Jugendfilmzentrum in Deutschland (Hg.) 2004: Kinder Film Kultur - Medienarbeit für Kinder und Jugendliche. Sonderdruck der "Kinder- und Jugendfilm Korrespondenz" anlässlich 25 Jahre Kinderkino München e.V. (Download: http://www.kjk-muenchen.de/ archiv/sonderdrucke.php)

Koebner, Thomas (Hg) 2007: Reclams Sachlexikon des Films. Reclam

Korte, Helmut 2004: Einführung in die Systematische Filmanalyse. Erich Schmidt Verlag, Berlin (3. überarbeitete und erweiterte Auflage).

Kuchenbuch, Thomas 1978: Filmanalyse. Theorien, Modelle, Kritik. Prometh Verlag, Köln.

Kümmerling-Meibauer/Koebner, Thomas 2010: Filmgenres. Kinder- und Jugendfilm. Reclams Universal Bibliothek 18728

Kurwinkel, Tobias/Schmerheim, Philipp (Hg.) 2013: Kinderund Jugendfilmanalyse. UVK Verlagsgesellschaft. Konstanz.

Mikos, Lothar 2003: Film- und Fernsehanalyse. UVK Verlagsgesellschaft, Konstanz.

Monaco, J. 2000: Film verstehen. Kunst, Technik, Sprache, Geschichte und Theorie des Films und der Medien. Mit einer Einführung in Multimedia. Rowohlt, Reinbek.

Pleyer, Peter 1974: Informationen zur Massenkommunikationslehre. Verlag C.J. Fahle, Münster.

Salber, Wilhelm 1977: Wirkungsanalyse des Films. Verlag Walter König, Köln.

Schäfer, Horst (Hg.) ab 1998: Lexikon des Kinder- und Jugendfilms im Kino, im Fernsehen und auf Video. CorianVerlag, Meitingen (Loseblattsammlung). 
Schäfer, Horst/Baacke, Dieter 1994: Leben wie im Kino. Jugendkulturen und Film. Fischer Verlag, Frankfurt/M.

Schnell, Ralf 2000: Medienästhetik. Zu Geschichte und Theorie audiovisueller Wahrnehmungsformen. Metzler, Stuttgart/ Weimar.

Strobel, Hans (Hg.) 1995: Inspiration Kinderfilm. Modelle der medienpädagogischen Vor- und Nachbereitung. Sonderdruck der "Kinder- und Jugendfilm Korrespondenz", hgg. von Kinderkino München e.V./Kinder- und Jugendfilmzentrum in Deutschland. München. (http://www.kjk-muenchen.de/ archiv/sonderdrucke.php)

Vollbrecht, Ralf 2001: Einführung in die Medienpädagogik. Beltz Verlag, Weinheim und Basel.

\section{DVD}

Werner Barg/Horst Schäfer 2005: Filmsprache und Filmanalyse. dffb/KJF. Auslieferung über den KJF-Medienvertrieb.

Deutsche Filmakademie 2011: Faszination Film. Vom Drehbuch bis zur Premiere. Genauere Einzelheiten: vierundzwanzig.de (Das Wissensportal der Deutschen Filmakademie). 